\title{
Success in recall as a function of success in implementation of mnemonic instructions
}

\section{P. S. DELIN, PSYCHOLOGY DEPARTMENT, UNIVERSITY OF ADELAIDE, South Australia}

The Ss wrote descriptions of bizarre images constructed to link pairs of nouns. These descriptions were assigned ratings for conformity to mnemonic instructions. In a recall test 15 weeks later performance was found to be related to the previously assigned ratings. There was some suggestion in the data that difference in interitem association among the word pairs affected the ability of the Ss to conform to the instructions. The Ss were not warned when the images were constructed that they would be expected to recall the material.

A number of researchers, e.g., Smith \& Noble (1965), Bugelski, Kidd, \& Segmen (1968), have found differences in learning performance between Ss given mnemonic instructions and control Ss. In no case, however, was it demonstrated that it was the carrying out of the instructions that mediated the differences in performance.

Similarly, it has been shown (e.g., Martin, Boersma, \& Cox, $1965)$, that the reporting of associative strategies is related to success in recall. It has not, however, been shown that the associative strategy is responsible for the correct recall.

The present study seeks to show, first, that qualitative differences in the image the $S$ makes affect his ability to recall the material, and, second, that differences in interitem association affect the S's ability to carry out specific mnemonic instructions. Method

Three pairs of items were used. These were selected from a large collection of item pairs which had previously been rated by 20 judges on a 5 point scale, for degree of interitem association. The item pairs selected, window-ladder, torch-handbag, and egg-piano had been given high $(\mathrm{H})$, medium $(\mathrm{M})$, and low $(\mathrm{L})$ mean ratings of $3.70,2.25$, and 1.05 , respectively.

The Ss were a class of 1072 nd year psychology students. The experimental sessions consisted of the opening few minutes of two of the regular lecture periods for this course, so it was only possible to analyze the results of the 88 students who were present at both sessions.

In the first session the experimenter announced that he was collecting a sample of bizarre images. He said that he was going to present pairs of words and requested the students to make images connecting them which were as active and bizarre as possible, and which made use of as many sensory modalities as possible. The students were to write descriptions of their images on the answer-sheets provided. The experimenter read two sample descriptions of images of the kind required, and the answer-sheets were distributed. The experimenter then presented the three pairs of words verbally, in the order $\mathrm{L}-\mathrm{H}-\mathrm{M}$, allowing $3 \mathrm{~min}$ for each pair. The students were then asked to write their names on the sheets, on the excuse that it might be necessary to collect a further sample from some Ss, and the answer-sheets were collected.

The descriptions were rated by the experimenter and one other judge on a 5 point scale for the degree to which they conformed to the instructions. The ratings of the judges were intercorrelated, giving a rho of .793 . Since this represented a reasonably high level of reliability, each image description was assigned a "goodness" rating equal to the mean of the ratings given by the two judges.

In the second session, which took place 15 weeks after the first, the experimenter reminded the students of what had taken place at the first session, explained that he was going to ask them to try to recall the material, and asked them to write their answers on a sheet of paper, ensuring that their neighbors did not see what they wrote. He then read the first word of each pair, in the same order as before, allowing $20 \mathrm{sec}$ for a response to each. The Ss wrote their names on their answer-sheets, which were then collected. Results

The results are summarized in Table 1. The mean ratings for those Ss who did and did not recall the items suggest that the ratings are positively related to recall performance. Mann-Whitney $\mathrm{U}$ tests corrected for ties and converted to normal deviates gave, for $L, z=2.11(p<.05)$, for $H, z=1.77(p=.08)$, and for $M, z=$ $2.82(\mathrm{p}<.01)$. These results, then, strongly suggest that the ratings relate to recall performance.

A Friedman two way analysis of variance carried out on the ratings for the three item pairs was highly significant $(p<.001)$, suggesting that $\mathrm{Ss}$ tended to make images of different degrees of "goodness" on the three item pairs. Wilcoxon signed-ranks matched-pairs tests carried out on the comparisons $L / H$ and $H / M$, with $\mathrm{T}$ converted to normal deviates produced, for $\mathrm{L} / \mathrm{H}, \mathrm{z}=3.28$ $(\mathrm{p}<.001)$, and for $\mathrm{H} / \mathrm{M}, \mathrm{z}=2.73(\mathrm{p}<.01)$. Thus each item pair produced a significantly different mean rating.

In order to compare the levels of recall performance on the three item pairs McNemar tests were carried out comparing $L / H$, $\mathrm{L} / \mathrm{M}$ and $\mathrm{H} / \mathrm{M}$. As the numbers involved in the $\mathrm{L} / \mathrm{H}$ comparison were small, this was evaluated by means of a Sign test which yielded $\mathrm{p}<.01$ (two tailed). The other two comparisons gave $\chi^{2}=$ $13.78(\mathrm{~L} / \mathrm{M}, \mathrm{p}<.001)$ and $\chi^{2}=22.40(\mathrm{H} / \mathrm{M}, \mathrm{p}<.001)$. Thus all three comparisons were significant, but it is worthy of note that the $\mathrm{L} / \mathrm{H}$ difference is in the direction opposite from that of the difference in "goodness" ratings between $\mathrm{L}$ and $\mathrm{H}$. Discussion

The results are highly consistent with the view that imagery mnemonics have a direct effect on performance; an effect which cannot be accounted for in terms of such factors as motivation. The Ss in this study did not know when they made their images that they would have to recall the material, and the ratings of the images were made some 10 weeks before the recall task, so the question of contamination does not arise.

Since one of the rating criteria was the level of bizarreness of the imagery the results are in sharp contrast to those of Wood (1966), who was unable to show that either imagery or bizarreness had any effect. However, there was no independent evidence that Wood's Ss produced any bizarre imagery, or indeed, any imagery at all. Laboratory experience would have led the present writer to predict that under the conditions used by Wood little bizarre imagery would be produced.

The differences between the ratings for the images produced for the different items are not quite as convincing. It is possible that content differences in the images could have led the judges to rate inconsistently in such a way as to produce these differences. On the other hand, the factors which would lead to this inconsistency are the same ones which might be expected to lead the Ss to make images of different degrees of "goodness" for the three item pairs. Although the interitem association values have high face validity it could be argued that it was not these which produced the observed differences in the images described. If this was correct our second prediction could not be regarded as having been clearly confirmed, but it would still be possible to maintain a less specific but nevertheless important position, namely that the ability of the Ss to carry out the instructions is affected by the nature of the item. And, by extension, this would lead to the reasonable hypothesis that the nature of the items will determine to some extent what types of natural language mediators will be used by uninstructed Ss.

Table 1

Mean "Goodness" Ratings and Numbers of Ss Succeeding and Not Succeeding in Recalling each Item

\begin{tabular}{|c|c|c|c|c|c|c|}
\hline & \multicolumn{2}{|c|}{ L } & \multicolumn{2}{|c|}{$\mathbf{H}$} & \multicolumn{2}{|c|}{ M } \\
\hline & Number & Rating & Number & Rating & Number & Rating \\
\hline $\begin{array}{r}\text { Correct } \\
\text { recall }\end{array}$ & 67 & 3.69 & 76 & 3.30 & 45 & 3.22 \\
\hline $\begin{array}{l}\text { Not correct } \\
\text { recall }\end{array}$ & 21 & 3.33 & 12 & 2.75 & 43 & 2.56 \\
\hline Total & 88 & 3.61 & 88 & 3.23 & 88 & 2.90 \\
\hline
\end{tabular}


The fact that the apparent relation between interitem association and "goodness" of image is not monotonic is not surprising. With low interitem association practically any image would be bizarre. With high interitem association, the form of the association is usually fairly specific, and hence easy to ignore. But with moderate interitem association there is usually a large number of ways in which the items could be related, and it might be difficult to put them all aside in favor of a bizarre one. Irrespective of such ad hoc theorizing there is no particular reason to predict a monotonic relation.

The reversal of direction of the differences between the ratings and levels of performance between $L$ and $H$ is interesting in view of the fact that for both of the groups ratings were related to successful recall. This relation was, however, not as clear for $\mathrm{H}$ as for $\mathrm{L}$, and this, together with the fact that the ratings for $\mathrm{H}$ showed higher variability (not significantly) than did the ratings for $L$, prompts the suggestion that some Ss did not succeed in putting aside the high pre-existing association between "window" and "ladder" in favor of a bizarre one, but that for some of these
Ss that pre-existing association was sufficient to mediate recall. Thus the difference in performance between $\mathrm{H}$ and $\mathrm{M}$ might partly depend on a difference between the potential of high and medium interitem associations for mediating recall in the absence of mnemonic images.

It is clear that it would be worthwhile to repeat this experiment using more items, and counterbalancing the orders in which pairs of different degrees of interitem association were presented.

\section{REFERENCES}

BUGELSKI, B. R., KIDD, E., \& SEGMEN, J. Image as a mediator in one-trial paired-associate learning. J. exp. Psychol, 1968, 76, 69-73.

MARTIN, C. J., BOERSMA, F. J., \& COX, D. L. A classification of associative strategies in paired-associate learning. Psychon. Sci., 1965, 3, 455-456.

SMITH, K., \& NOBLE, C. E. Effects of a mnemonic technique applied to verbal learning and memory. Percept. mot. Skills, 1965, 21, 123-134.

woOD, G. Mnemonic studies in recall. Ph. D. Dissertation, Northwestern University, 1966. 